$\mathcal{B} \mathcal{T} \mathcal{P} \mathcal{S}$

Brazilian Transportation

Planning Society
Journal of Transport Literature

Vol. 7, n. 4, pp. 130-146, Oct. 2013

Research Directory

\section{JTL | RELIT}

www.transport-literature.org ISSN 2238-1031

\title{
Análise da modelagem da escolha aeroportuária em regiões de múltiplos aeroportos
}

[Analysis of airport choice modeling in multi-airport regions]

\author{
Mayara Condé Rocha Murça*, Anderson Ribeiro Correia \\ Instituto Tecnológico de Aeronáutica - Brasil
}

Submitted 4 Apr 2012; received in revised form 30 Jun 2012; accepted 1 Oct 2013

\begin{abstract}
Resumo
A análise da competição em sistemas de múltiplos aeroportos representa um tema bastante discutido na literatura, em função de sua importância para o planejamento aeroportuário e das companhias aéreas. Faz-se necessário compreender o conjunto de atributos de nível de serviço considerados pelo usuário de transporte aéreo no momento da decisão por um aeroporto, a fim de capturar e prever o seu comportamento diante de alternativas. Em geral, o tempo de acesso ao aeroporto, a frequência de voo e a tarifa representam as variáveis determinantes da escolha aeroportuária, mas com diferentes graus de importância relativa, dependendo da região e do cluster analisado. Assim, este trabalho tem o objetivo de analisar a evolução de estudos que visam simular a escolha do passageiro em regiões de múltiplos aeroportos, abordando as diferenças das principais metodologias empregadas e dos resultados obtidos.
\end{abstract}

Palavras-Chave: múltiplos aeroportos, escolha, passageiro, planejamento.

\section{Abstract}

The analysis of multi-airport systems' competition is a topic widely discussed in the literature due to its importance for airports and airlines planning. It's necessary to understand the set of attributes of level of service considered by the air transportation user at the time of airport choice in order to capture and predict his behavior when dealing with alternative. In general, access time to the airport, flight frequency and price represent the determinants variables of airport choice, but with different degrees of relative importance, depending on the region and the cluster in analysis. Thus, this work intend to analyze the evolution of studies designed to simulate the passenger's choice in multi-airport regions, addressing the differences between main methods used and results obtained.

Key words: multi-airport, choice, passenger, planning.

*Email: may.crm@gmail.com.

\section{Recommended Citation}

Murça, M. C. R. and Correia, A. R. (2013) Análise da modelagem da escolha aeroportuária em regiões de múltiplos aeroportos. Journal of Transport Literature, vol. 7, n. 4, pp. 130-146.

- JTL/RELIT is a fully electronic, peer-reviewed, open access, international journal focused on emerging transport markets and published by BPTS - Brazilian Transport Planning Society. Website www.transport-literature.org. ISSN 2238-1031. 


\section{Introdução}

Este trabalho consiste na realização de uma análise crítica sobre a evolução da modelagem da escolha aeroportuária e de seus condicionantes em regiões de múltiplos aeroportos. Este tema vem sendo estudado há décadas em diversas regiões do globo e ainda apresenta grande destaque no mundo científico atualmente. Isso se deve ao fato de que a análise da competição entre aeroportos e sistemas de aeroportos é um assunto de vital importância para o planejamento de investimentos e, portanto, para os administradores aeroportuários e de empresas aéreas.

Esta necessidade de entendimento do ambiente competitivo, especialmente em sistemas de múltiplos aeroportos, está associada a uma série de fatores. A definição da área de influência de cada aeroporto, por exemplo, torna-se muito mais complexa, uma vez que há concorrência direta entre alternativas de voos de companhias aéreas, níveis de serviço das instalações, modos de acessibilidade etc. A competição também eleva o nível de complexidade na projeção de demanda, a fim de que não ocorra a omissão de variáveis explicativas relevantes, e na regulação do mercado, no que tange à análise do poder de mercado de companhias aéreas e a questões práticas, como, por exemplo, a alocação de slots. Assim, faz-se necessário desenvolver ferramentas eficientes que permitam compreender e capturar o comportamento dos usuários do transporte aéreo no que diz respeito às suas escolhas diante de diferentes cenários característicos do ambiente competitivo.

Atualmente, grande parte das regiões metropolitanas do mundo conta com mais de um aeroporto comercial para atender o elevado contingente populacional e suas necessidades crescentes de transporte. Segundo De Luca (2012), podem ser identificados 59 sistemas de múltiplos aeroportos hoje, sendo 25 na Europa, 18 na América do Norte, 8 na Ásia, 5 na América Latina e 3 no Oriente Médio. Alguns deles já se encontram bem consolidados, como Nova Iorque, Londres e Tóquio, ao passo que outros tem assumido um papel cada vez mais importante no mercado de aviação internacional, como Seul, Bangkok e Manchester. Ainda, verifica-se que sistemas de um único aeroporto estão em fase de transição para sistemas de múltiplos aeroportos, como é o caso de Johannesburgo, Beijing e Lisboa. Dentre os fatores que tem gerado este crescimento acelerado, pode-se citar: globalização, privatização, atuação 
de companhias aéreas low-cost, reorganização de empresas aéreas tradicionais, melhorias na acessibilidade aos aeroportos, aumento da importância de aeroportos regionais e inovação tecnológica em aeronaves (De Luca, 2012).

A maioria dos estudos presentes na literatura utilizam modelos de escolha discreta baseados na teoria da utilidade para modelar o comportamento do usuário no que tange à escolha de uma alternativa. No caso do transporte aéreo, diversos tipos de modelos tem sido desenvolvidos para simular a escolha do passageiro por um determinado aeroporto e até mesmo por combinações de duas ou mais dimensões, envolvendo não apenas o aeroporto, mas também a companhia aérea (Ishii et al, 2009) e o meio de transporte para acesso (Pels et al, 2003). É importante observar que um voo é o resultado de um conjunto de escolhas realizadas pelo usuário de transporte aéreo, as quais englobam desde a decisão de viajar, até o destino final da viagem, passando pela escolha de aeroporto de embarque, companhia aérea, horário e duração da viagem, tarifa e modal de acesso (Harvey, 1987). Desta forma, o estudo da interação dessas dimensões de escolha é fundamental para que o problema seja tratado de maneira mais realista.

Apesar das diferentes modelagens matemáticas e decisão de inclusão ou não de determinadas dimensões de escolha, a maioria dos estudos aponta que as principais variáveis condicionantes da escolha aeroportuária são o tempo de acesso, a frequência de voo e a tarifa, sendo as duas primeiras mais importantes. Além disso, os resultados revelam que a segmentação em clusters é recomendável, uma vez que, em muitos casos, aparecem diferenças comportamentais significativas entre os mesmos, sobretudo quando a segmentação está relacionada ao motivo da viagem.

Em síntese, a competição em regiões de múltiplos aeroportos tem sido analisada principalmente através de metodologias que envolvem duas etapas fundamentais: coleta de dados de preferência com passageiros usuários do sistema e modelagem matemática da escolha aeroportuária, a partir da base de dados obtida, utilizando modelos de escolha discreta. Para contemplar todos os aspectos relacionados ao tema e à metodologia supracitada e, assim, realizar uma ampla revisão da literatura, este trabalho foi assim dividido: na Seção 1, apresenta-se a teoria que embasa os modelos de escolha discreta, os quais são apresentados na Seção 2; na Seção 3, abordam-se as técnicas de coleta de dados utilizados na calibração dos 
modelos e, na Seção 4, é feita uma análise comparativa sobre alguns estudos que focam este tema, sobretudo no que diz respeito aos principais resultados obtidos.

\section{A teoria da utilidade}

O método mais empregado para simulação do comportamento de um indivíduo diante de um conjunto de alternativas tem sido os modelos de escolha discreta, os quais se sustentam na teoria da utilidade. Esta teoria baseia-se na hipótese de que cada indivíduo é um ser racional que realiza suas escolhas com o objetivo de maximização da utilidade (Edwards, 1954). Assumindo que o usuário de transporte aéreo seja modelado como um "tomador de decisão" racional, sua escolha aeroportuária será realizada de forma a maximizar a utilidade proveniente de um conjunto de atributos.

Considere-se que o decisor tenha um portfólio $I$ de alternativas independentes. Para cada alternativa $j$ de $I$, o decisor atribui uma utilidade $U_{j}$, a qual depende de um conjunto de atributos (características mensuráveis) da alternativa e do próprio tomador de decisão. Assim, a utilidade $U_{j}$ pode ser entendida como $U\left(X_{j}\right)$, sendo $X_{j}$ o vetor de atributos relacionados à alternativa $j$ e ao tomador de decisão. A probabilidade de escolha da alternativa $j$ dentre as disponíveis no portfólio I é dada pela Equação (1).

$$
p[j / I]=P_{r}\left[U_{j}>U_{k}, \forall k \neq j, k \in I\right]
$$

Por sua vez, a utilidade $U_{j}$ pode ser expressa pela soma de uma componente sistemática $V_{j}$ com uma componente aleatória $\varepsilon_{j}$ (Walker e Ben-Akiva, 2002), como mostra a Equação (2). A existência de uma componente aleatória $\varepsilon_{j}$ está associada ao fato de que o processo de escolha envolve fatores subjetivos que não podem ser internalizados pelo investigador, representando assim, um desvio desconhecido da utilidade sistemática $V_{j}$ (média dos valores esperados de utilidade percebida pelo tomador de decisão), a qual pode ser estimada pelo analista. Por sua vez, a utilidade sistemática $V_{j}$ pode ser expressa como uma função dos atributos relativos à alternativa $j$ e ao tomador de decisão. Por conveniência estatística e analítica, assume-se que $V_{j}$ é uma função linear dos atributos $X_{k j}$ ou de suas transformadas funcionais $f\left(X_{k j}\right)$, como mostra a Equação (3). Para o caso do transporte aéreo, a escolha aeroportuária depende de atributos do indivíduo e do aeroporto, tais como: data da viagem e 
hora do voo, modal de acesso, preço da passagem aérea, disponibilidade de voos, companhia aérea, experiência com o aeroporto, dentre outros (Harvey, 1987).

$$
\begin{gathered}
U_{j}=V_{j}+\varepsilon_{j} \\
V_{j}=\sum_{k} \beta_{k} \cdot X_{k j}+\sum_{q} \beta_{q} \cdot f\left(X_{q j}\right)
\end{gathered}
$$

A partir da hipótese central da teoria da utilidade, vários modelos de escolha discreta são desenvolvidos e diferem no que diz respeito à função de distribuição de probabilidade adotada para a componente aleatória $\varepsilon_{j}$ da utilidade.

\section{Modelos de escolha discreta}

A utilidade associada à uma alternativa engloba uma componente sistemática e outra aleatória, a qual pode assumir diferentes funções de distribuição de probabilidade, caracterizando um modelo de escolha.

O logit multinomial é um dos modelos mais simples de escolha discreta e também o mais utilizado (Harvey, 1987; Basar e Bhat, 2004; Loo, 2008; De Luca, 2012). Ele se baseia na hipótese de que os resíduos $\varepsilon_{j}$ da função utilidade são identicamente e independentemente distribuídos conforme uma distribuição de Gumbel. Sabendo-se que a diferença entre duas distribuições de Gumbel resulta em uma distribuição logística, a probabilidade de escolha da alternativa $j$ dentre as disponíveis no portfólio $I$ de opções é expressa pela Equação (6), a qual pode ser obtida a partir das Equações (4) e (5) que consideram apenas duas alternativas. A popularidade do logit está relacionada à sua facilidade de manuseio analítico, evidenciada, por exemplo, na extração de elasticidades. A Equação (7) apresenta a expressão da elasticidade para este modelo, representada pela variação da probabilidade $p[j]$ em função da variação de um atributo $X_{k j}$ da alternativa $j$.

$$
p[j]=P\left(U_{j} \geq U_{k}\right)=\int_{-\infty}^{\varepsilon_{n}} f\left(\varepsilon_{n}\right)=\int_{-\infty}^{\varepsilon_{n}} \frac{\mu e^{-\mu \varepsilon_{n}}}{\left(1+e^{-\mu \varepsilon_{n}}\right)^{2}} d \varepsilon_{n}=\frac{1}{1+e^{-\mu \varepsilon_{n}}}
$$




$$
\begin{gathered}
p[j]=\frac{1}{1+e^{-\left(V_{j}-V_{k}\right)}}=\frac{1}{1+e^{-\left(V_{j}-V_{k}\right)}} \frac{e^{V_{j}}}{e^{V_{j}}}=\frac{e^{V_{j}}}{e^{V_{j}}+e^{V_{k}}} \\
p[j]=\frac{e^{V_{j}}}{\sum_{m \in I} e^{V_{m}}} \\
\eta_{k j}=\beta_{k} X_{k j}(1-p[j])
\end{gathered}
$$

Algumas variações deste modelo e que foram utilizadas em estudos de sistemas de múltiplos aeroportos incluem o logit hierárquico (Pels et al, 2003; De Luca, 2012) e o logit multinomial misto (De Luca, 2012). O modelo logit hierárquico (ou aninhado) é utilizado quando se verifica a existência de correlação entre as alternativas, ou seja, a hipótese de independência dos resíduos $\varepsilon_{j}$ é relaxada. Assim, as alternativas com características comuns são agrupadas em conjuntos mutuamente excludentes e são criados níveis hierárquicos. Nesse caso, a probabilidade de escolha pode ser obtida pela multiplicação entre duas probabilidades oriundas do modelo logit puro. Já o modelo logit multinomial misto caracteriza-se pela segmentação da componente aleatória $\varepsilon_{j}$ em duas partes, uma delas caracterizada por independência entre alternativas e indivíduos e outra caracterizada por existência de correlação (Hensher e Greene, 2003). A distribuição da parte não independente da componente residual depende de parâmetros e dados observáveis relacionados ao tomador de decisão e às alternativas.

Apesar de pouco visto na literatura (Blackstone et al, 2006) quando se estuda o tema em questão, um outro modelo de escolha discreta utilizado para simular o comportamento do usuário durante a escolha de aeroportos é o probit. Ele se baseia na hipótese de que os resíduos $\varepsilon_{j}$ da função utilidade assumem uma distribuição normal com média $\mu$ e desvio padrão $\sigma$, resultando na probabilidade expressa pela Equação (8). Como se pode perceber, o probit não pode ser expresso em uma forma fechada como o logit, o que inibe seu manuseio analítico e a extração de elasticidades, as quais devem ser obtidas por métodos numéricos.

$$
P=\frac{1}{\sqrt{2 \pi}} \int_{-\infty}^{(x-\mu) / \sigma} e^{-x^{2} / 2} d x
$$




\section{Técnicas de coleta de dados}

Para a análise das preferências do passageiro em regiões de múltiplos aeroportos, faz-se necessário obter uma base de dados que permita extrair uma amostra representativa da população estudada e, assim, viabilizar toda a modelagem matemática. A maioria das técnicas para coleta de dados baseia-se em métodos de pesquisa de preferência declarada e revelada com usuários (Hensher et al, 1998).

O método de preferência revelada é utilizado quando se deseja capturar efetivamente o comportamento do usuário no sistema de transportes. A coleta de dados é feita, em geral, através de questionários com questões diretas sobre a situação em que o indivíduo se encontra no momento da pesquisa. É válido observar que a preferência revelada também pode ser avaliada por dados estatísticos provenientes de observação direta do comportamento decisório, sem a necessidade de questionários. Dentre as limitações deste método, destaca-se o fato de o mesmo não viabilizar a percepção de atributos rejeitados pelo indivíduo e a avaliação comportamental diante de opções não existentes. Além disso, a preferência revelada pode se tornar inútil em uma análise de escolha se a mesma foi realizada sem lastro comportamental.

Por outro lado, o método de preferência declarada procura capturar a preferência do usuário a partir de suas escolhas em um experimento decisório composto por alternativas preestabelecidas com atributos diversos decompostos em níveis. Assim, é possível avaliar o comportamento do indivíduo diante de cenários hipotéticos. A principal crítica a este método está relacionada a diferenças comportamentais observadas quando da transformação do cenário hipotético em realidade, visto que, na prática, o indivíduo pode não agir conforme havia indicado na pesquisa devido a uma série de fatores, tais como grau de detalhamento da pesquisa, contexto momentâneo, seleção de assuntos, restrições de horizonte de tempo e conjuntos de escolhas (Carlsson, 2010). O maior exemplo disto é conhecido na literatura como o problema do viés hipotético das pesquisas de preferência declarada (Loomis, 2011). Ele resulta quando os respondentes reportam uma disposição a pagar maior do que aquela observada quando eles são submetidos a condições reais. O indivíduo, em geral, conhece apenas as alternativas que estão à sua disposição e, em virtude da incerteza da alternativa hipotética, pode tender a exagerar no atributo novo oferecido, superestimando o bem estar que 
teria com isso. Um dos motivos desta incerteza pode ser a dificuldade de antecipação dos potenciais problemas da nova alternativa, os quais poderiam reduzir a disposição a pagar do indivíduo pela mesma.

Não há uma teoria geral amplamente aceita sobre o comportamento do entrevistado que explique a magnitude do viés hipotético como função das características da pesquisa, bem como as causas deste problema. Alguns estudos sugerem intervenções no formato da pesquisa, para reduzir a ocorrência do viés nas respostas, e outros sugerem intervenções após a pesquisa, para corrigir os resultados obtidos. No primeiro caso, as seguintes estratégias já foram investigadas: lançar a pesquisa como um referendo de assessoria à tomada de decisão de um órgão público sobre uma política realista; dizer ao entrevistado que pesquisas anteriores mostraram que os respondentes exageraram em sua disposição a pagar e instruí-lo para não fazer o mesmo; informar ao entrevistado as regras de provisão do bem hipotético (Loomis, 2011). No segundo caso, tem-se como exemplo as seguintes abordagens: criar uma escala de certeza da resposta durante a pesquisa e introduzir os resultados na análise estatística da disposição a pagar; reduzir a disposição a pagar hipotética por fatores empíricos ou obtidos a partir de meta-análises (Loomis, 2011). Apesar disso, nenhum estudo conseguiu explicar as causas do viés hipotético nem tratá-lo sem limitações.

Também motivados pelo fato de os experimentos tradicionais de pesquisa de preferência declarada simularem decisões que, muitas vezes, não traduzem a realidade dos mercados, Collins et al (2012) investigaram a utilização de uma abordagem interativa para o método de preferência declarada no estudo do comportamento do usuário de transporte aéreo. Uma vez que a internet tem se transformado em uma ferramenta bastante utilizada para a tomada de decisões no mundo atual, Collins et al (2012) desenvolveram um experimento online de escolha com o objetivo de aumentar o realismo desse tipo de pesquisa. Assim, foi criada uma agência de viagens online, onde o indivíduo era apresentado a diversas opções de passagens aéreas. Dentre as funcionalidades da plataforma interativa, era possível reordenar ou remover opções e ocultar ou exibir atributos. Ao mesmo tempo, foram utilizados os métodos de pesquisa convencionais para fins de comparação. Collins et al (2012) concluíram que o método interativo aumenta o envolvimento do indivíduo com este tipo de experimento, além de gerar dados que conduzem a modelos com maior desempenho. 
Apesar das limitações anteriormente expostas, uma das vantagens do método de preferência declarada em relação ao de preferência revelada consiste na obtenção de maior quantidade de informação por indivíduo, o que pode reduzir o custo da pesquisa. Além disso, ele permite obter modelos com maior estabilidade no tempo e no espaço e que caracterizam os trade-offs enfrentados pelo indivíduo.

\section{Análise comparativa de estudos sobre sistemas de múltiplos aeroportos}

Após a discussão sobre as principais características de métodos de coleta de dados e modelos matemáticos utilizados para simular a escolha do passageiro em regiões de múltiplos aeroportos, realizou-se uma análise comparativa de alguns estudos nesse tema, abrangendo diferentes regiões do globo e tempos variados, sobretudo no que diz respeito aos resultados encontrados. Na Tabela 1, são apresentados detalhes desses estudos quanto à região estudada, método utilizado para coleta de dados e modelo matemático. Em seguida, são mostrados os diferentes resultados obtidos para cada sistema de múltiplos aeroportos considerado, os quais são confrontados e comparados entre si.

Tabela 1 - Estudos sobre escolha aeroportuária em regiões de múltiplos aeroportos ${ }^{1}$

\begin{tabular}{|c|c|c|c|c|}
\hline Pesquisador & Área estudada & $\begin{array}{l}\text { Número de } \\
\text { entrevistados }\end{array}$ & $\begin{array}{l}\text { Método de coleta } \\
\text { de dados }\end{array}$ & Modelo matemático \\
\hline $\begin{array}{l}\text { Harvey } \\
(1987)\end{array}$ & $\begin{array}{l}\text { Área da Baía de } \\
\text { São Francisco }\end{array}$ & 2800 & $\begin{array}{l}\text { Preferência } \\
\text { revelada }\end{array}$ & Logit multinomial \\
\hline $\begin{array}{l}\text { Pels et al } \\
(2003)\end{array}$ & $\begin{array}{l}\text { Área da Baía de } \\
\text { São Francisco }\end{array}$ & 21500 & $\begin{array}{l}\text { Preferência } \\
\text { revelada }\end{array}$ & Logit aninhado \\
\hline $\begin{array}{l}\text { Basar e Bhat } \\
\text { (2004) }\end{array}$ & $\begin{array}{l}\text { Área da Baía de } \\
\text { São Francisco }\end{array}$ & 1918 & $\begin{array}{l}\text { Preferência } \\
\text { revelada }\end{array}$ & $\begin{array}{l}\text { Logit multinomial com } \\
\text { formação probabilística de } \\
\text { painel de alternativas }\end{array}$ \\
\hline $\begin{array}{l}\text { Blackstone } \\
\text { et al (2006) }\end{array}$ & $\begin{array}{l}\text { Região do Médio } \\
\text { Atlântico }\end{array}$ & 1100 & $\begin{array}{l}\text { Preferência } \\
\text { revelada (por } \\
\text { telefone) }\end{array}$ & Probit \\
\hline Loo (2008) & $\begin{array}{l}\text { Delta do Rio das } \\
\text { Pérolas (Hong } \\
\text { Kong) }\end{array}$ & 308 & $\begin{array}{l}\text { Preferência } \\
\text { declarada }\end{array}$ & Logit multinomial \\
\hline $\begin{array}{l}\text { Ishii et al } \\
(2009)\end{array}$ & $\begin{array}{l}\text { Área da Baía de } \\
\text { São Francisco }\end{array}$ & 1752 & $\begin{array}{l}\text { Preferência } \\
\text { revelada }\end{array}$ & $\begin{array}{c}\text { Logit condicional } \\
\text { ponderado e logit misto } \\
\text { ponderado }\end{array}$ \\
\hline $\begin{array}{l}\text { De Luca } \\
\text { (2012) }\end{array}$ & $\begin{array}{c}\text { Região de } \\
\text { Campanha (Itália) }\end{array}$ & 800 & $\begin{array}{l}\text { Preferência } \\
\text { declarada }\end{array}$ & $\begin{array}{l}\text { Logit multinomial, logit } \\
\text { hierárquico e logit } \\
\text { multinomial misto }\end{array}$ \\
\hline
\end{tabular}

\footnotetext{
${ }^{1}$ Fonte: autores, com base em levantamento bibliográfico.
} 


\subsection{Análise dos resultados obtidos para cada região estudada}

\subsection{1 Área da Baía de São Francisco}

Dentre todas as regiões de múltiplos aeroportos no mundo, a Área da Baía de São Francisco, a qual compreende os aeroportos San Francisco International (SFO), Oakland International (OAK) e San Jose Municipal (SJC), tem sido a mais estudada.

Harvey (1987) investigou as características da escolha aeroportuária para residentes desta região a partir de dados de uma pesquisa realizada com passageiros em 1980. Para isso, utilizou modelos logit multinomial considerando apenas dois clusters: passageiros (residentes) a negócio ou não. Segundo Harvey (1987), faz-se necessário segmentar os usuários em clusters, uma vez que podem aparecer diferenças comportamentais significativas entre os mesmos. Por exemplo, passageiros residentes na região investigada possuem informações mais completas sobre as características de aeroportos disponíveis, como padrão de tráfego durante acesso, quando comparados aos não residentes, o que faz com que algumas variáveis influenciem a decisão em grau diferente. Ainda, passageiros a negócio são menos elásticos a preço e mais elásticos ao tempo de viagem e à frequência de voo quando comparados aos passageiros que não viajam a negócio.

Dentre os resultados obtidos, tem-se que um modelo logit baseado nas variáveis "tempo de acesso ao aeroporto" e "frequência de voos para o destino escolhido" forneceu uma boa aproximação para o comportamento do usuário quanto à escolha de aeroporto, sobretudo para passageiros a negócio. Ainda, Harvey (1987) concluiu que: voos diretos são preferíveis a voos com conexão, voos diretos sem escalas e voos diretos com escalas não apresentaram efeitos diferentes no comportamento do passageiro, o tempo de acesso se mostrou mais importante para voos de curta distância e passageiros a negócio são realmente mais sensíveis à frequência e ao tempo de acesso do que passageiros que não viajam a negócio.

Pels et al (2003), Basar e Bhat (2004) e Ishii et al (2009) também investigaram a Área da Baía de São Francisco. Pels et al (2003) utilizaram modelos logit aninhado para descrever o processo de escolha considerando duas dimensões: aeroporto e modal de acesso. As variáveis utilizadas foram tarifa, frequência, tempo e custo de acesso ao aeroporto. Assim como nos estudos de Harvey (1987), foram analisados apenas os passageiros residentes na região. A 
escolha da companhia aérea não foi incluída na análise devido à indisponibilidade de alguns dados de tarifa. Pels et al (2003) obtiveram que um modelo logit aninhado com a escolha do modal de acesso internalizada na escolha do aeroporto se mostrou mais adequado. Além disso, verificaram que o tempo de acesso é a variável explicativa dominante na escolha do aeroporto, sendo mais importante que o custo de acesso e que a elasticidade-tarifa apresentou valores relativamente pequenos. Logo, Pels et al (2003) concluíram que, para aumentar a participação no mercado, operadores aeroportuários e autoridades locais devem investir em modais de acesso mais rápidos e que um aumento nas tarifas pode ter pouco impacto na demanda, ao passo que investimentos na melhoria de qualidade do acesso trazem benefícios tanto para o aeroporto quanto para as empresas aéreas.

Ishii et al (2009) também consideraram duas dimensões para descrever o processo de escolha aeroportuária, mas substituíram o modal de acesso pela companhia aérea. Para o modelo logit condicional ponderado proposto, as variáveis mais importantes foram: tempo de acesso, tempo de espera no aeroporto, frequência de voo, disponibilidade de combinações específicas aeroporto-companhia aérea e a possibilidade de chegar cedo no destino. Ishii et al (2009) reforçaram que a escolha da empresa aérea não pode ser desconsiderada na análise de sistemas de múltiplos aeroportos, já que as decisões não são tomadas de forma independente.

Por fim, Basar e Bhat (2004) analisaram a escolha de aeroporto para passageiros a negócio residentes na Área da Baía de São Francisco e introduziram uma característica inovadora baseada no reconhecimento de que passageiros podem não considerar todos os aeroportos disponíveis, ou seja, aqueles que possuem pelo menos um voo (direto ou não) para o destino desejado, no momento em que realizam a escolha. Isso se deve ao fato de que o conjunto de alternativas formado por um indivíduo depende de aspectos sociodemográficos, psicológicos e informativos, como também de critérios associados às suas atitudes e percepções. Dessa forma, faz-se necessário obter este conjunto de opções considerado pelo passageiro, o qual é oriundo de suas percepções subjetivas, antes de analisar a escolha realizada. Para isso, Basar e Bhat (2004) utilizaram modelos probabilísticos de geração de conjunto de alternativas, com uma abordagem baseada em restrição, na qual um aeroporto é excluído das opções consideradas pelo passageiro caso sua utilidade seja menor do que um valor limite. Para a modelagem da escolha de aeroporto a partir da definição do conjunto de alternativas, Basar e Bhat (2004) optaram pelo familiar modelo logit multinomial e foram utilizadas variáveis 
relacionadas ao nível de serviço e às suas interações com aspectos sociodemográficos e características da viagem. Em seus resultados, Basar e Bhat (2004) corroboraram o fato de que o tempo de acesso e a frequência de voo são as variáveis determinantes na escolha de aeroporto, mas obtiveram variações na sensibilidade para diferentes características do viajante e do voo. O uso de um modelo probabilístico de formação do conjunto de alternativas prédecisão permitiu tecer considerações para diferentes tipos de indivíduos tanto no estágio de percepção de disponibilidade do aeroporto quanto de escolha propriamente dita, além de possibilitar uma superação do modelo logit multinomial puro na avaliação estatística dos dados.

\subsubsection{Campanha (Itália)}

Estimando diferentes modelos de escolha discreta, De Luca (2012) estudou como o tipo de conexão, a duração e a data da viagem afetam o comportamento decisório do usuário e investigou efeitos não lineares de acessibilidade e nível de serviço. A análise foi feita a partir de uma pesquisa de preferência declarada criada especificamente para o estudo e realizada com residentes na região da Campanha, sul da Itália, os quais contavam com as seguintes alternativas de aeroporto: Roma Fiumicino, Nápoles Capodichino e Roma Ciampino. De Luca (2012) utilizou três diferentes tipos de modelos de escolha discreta baseados na teoria da utilidade (logit multinomial, logit hierárquico e logit multinomial misto) para cenários de voos diretos, voos com conexão e diferentes planos de viagem e testou uma série de variáveis, tais como tarifa, frequência, duração do voo, tempo e distância de acesso, sexo e idade do passageiro etc. Dentre as principais conclusões obtidas, tem-se que a escolha de aeroporto é bastante influenciada pelo plano de viagem do passageiro e por sua experiência com viagens anteriores, que os modelos estimados apenas para voos diretos podem não simular efetivamente o comportamento do usuário se apenas voos com conexão estiverem disponíveis e que o familiar modelo logit multinomial continua sendo uma abordagem efetiva, apesar de os modelos logit multinomial misto e logit aninhado terem superado estatisticamente todos os outros modelos. Os resultados para voos diretos indicaram que nível de serviço e atributos socioeconômicos, como idade, experiência e renda, são estatisticamente significantes e que transformações não lineares para frequência de voo e tempo de acesso melhoraram a qualidade do ajuste. Ao contrário dos estudos realizados na Área da Baía de São Francisco, De Luca (2012) obteve que apenas o tempo de acesso pode alterar significativamente o 
market share do aeroporto, ou seja, a frequência de voo e a tarifa pouco influenciam. Para o cenário de voos com conexão, atributos socioeconômicos se mostraram pouco significantes, ao passo que a duração da viagem e a tarifa revelaram possuir o maior poder de alteração do market share do aeroporto.

\subsubsection{Médio Atlântico}

Blackstone et al (2006) investigaram a escolha de aeroporto na região do Médio Atlântico envolvendo os seguintes aeroportos: Baltimore-Washington Internacional, Newark Internacional, John F. Kennedy Internacional e Filadélfia Internacional. Para isso, desenvolveram pesquisas por telefone com a população da área de mercado do aeroporto internacional da Filadélfia. Através da estimação de modelos probit, Blackstone et al (2006) obtiveram que a renda, a experiência do passageiro, a facilidade de estacionamento, o tempo de acesso, a disponibilidade de tarifas mais baixas e de voos internacionais são variáveis significativas na escolha do aeroporto. É válido ressaltar que a pesquisa foi realizada com usuários de apenas um dos aeroportos da região, devendo-se ter cuidado na generalização dos resultados para todo o sistema.

\subsubsection{Delta do Rio das Pérolas}

Apesar da grande quantidade de estudos existente sobre sistemas de múltiplos aeroportos, verifica-se que estes estão concentrados em regiões específicas, como a área da Baía de São Francisco, do primeiro mundo ocidental. No entanto, é razoável imaginar que o conjunto de atributos de nível de serviço determinante da escolha aeroportuária possa variar significativamente em diferentes partes do globo, em função dos variados cenários culturais, políticos e econômicos existentes.

Com o objetivo de avaliar esta questão para o contexto asiático, Loo (2008) investigou a escolha aeroportuária de passageiros que partiam do aeroporto internacional de Hong Kong para quinze diferentes destinos. Apesar de a pesquisa ter sido realizada apenas com passageiros de um dos aeroportos da região do Delta do Rio das Pérolas, Loo (2008) afirma que os resultados conduzem a apontamentos interessantes sobre este sistema de múltiplos aeroportos, o qual foi muito pouco estudado até hoje. Através de pesquisas de preferência declarada e da utilização de um modelo logit multinomial, concluiu-se que, além do tempo de 
acesso e da frequência de voo, a tarifa e o número de companhias aéreas constituíram atributos de nível de serviço importantes, ao passo que a quantidade de modais para acessar o aeroporto e o custo do acesso representaram variáveis estatisticamente insignificantes. $\mathrm{O}$ restante dos resultados se mostrou coerente com os demais estudos realizados, como, por exemplo, o fato de passageiros a negócio serem mais sensíveis ao tempo de acesso e à frequência de voo e menos sensíveis à tarifa do que passageiros que não viajam a negócio.

\subsection{Análise comparativa de resultados obtidos}

A despeito da utilização de modelos de escolha discreta diversos e das diferentes características das regiões analisadas, a maioria dos estudos converge para o fato de que as variáveis determinantes da escolha aeroportuária são: tempo de acesso ao aeroporto, frequência de voo e tarifa. A Tabela 2 apresenta os resultados da estimação dos parâmetros associados a estas variáveis para cada estudo apresentado na Tabela 1. Estes resultados estão divididos por clusters relacionados ao motivo da viagem (negócio ou lazer) para os casos em que a segmentação foi realizada. Ainda, apresentam-se outras variáveis incluídas no modelo que se mostraram estatisticamente significantes, bem como a qualidade do ajuste, quando disponível.

Verificou-se que a tarifa e o tempo de acesso são negativamente correlacionados com a utilidade e que a frequência de voo é positivamente correlacionada com a utilidade em quase todos os casos. Apenas Blackstone et al (2006) obtiveram que a distância da residência até o aeroporto é positivamente correlacionada com a utilidade. Isso aconteceu devido à notável dificuldade de acesso do aeroporto analisado (John F. Kennedy) quando comparado aos demais e à possibilidade de passageiros situados em localidades mais distantes atribuírem maior peso a outros fatores em detrimento da acessibilidade, o que pode aumentar a utilidade do aeroporto com maior dificuldade de acesso. Em geral, o tempo de acesso e a frequência revelaram maior poder de influência na utilidade do que a tarifa.

No que diz respeito à segmentação em clusters associados ao motivo da viagem, observou-se que, na maioria dos estudos, a tarifa é mais importante para passageiros que não viajam a negócio, ao passo que o tempo de acesso e a frequência de voo são mais importantes para passageiros que viajam a negócio. 
Finalmente, verificou-se que, em geral, o modelo logit multinomial puro é uma abordagem satisfatória para a simulação do comportamento do passageiro e que a utilização de abordagens mais complexas traz um incremento pouco significante na qualidade do ajuste. No caso do estudo de De Luca (2012), por exemplo, o $\mathrm{R}^{2}$ foi de 0,351 para o modelo logit multinomial, de 0,353 para o logit hierárquico e de 0,36 para o logit multinomial misto, considerando as variáveis relacionadas a atributos socioeconômicos do passageiro, além de tempo de acesso, frequência e tarifa.

Tabela 2 - Resultados da estimação de parâmetros dos estudos analisados ${ }^{1}$

\begin{tabular}{|c|c|c|c|c|c|c|c|}
\hline \multicolumn{2}{|c|}{ Variáveis } & \multicolumn{2}{|c|}{ Tempo de acesso } & \multicolumn{2}{c|}{ Frequência } & \multicolumn{2}{c|}{ Tarifa } \\
\hline \multicolumn{1}{|c|}{ Motivo da viagem } & Negócio & Lazer & Negócio & Lazer & Negócio & Lazer \\
\hline \multirow{5}{*}{$\begin{array}{c}\text { Harvey } \\
(1987)^{2}\end{array}$} & $-0,166$ & $-0,12$ & 3,27 & 2,95 & - & - \\
\cline { 2 - 8 } & $\begin{array}{c}\text { Pels et al } \\
(2003)^{3}\end{array}$ & $-0,138$ & $-0,076$ & 1,353 & 1,358 & $-0,001$ & $-0,009$ \\
\cline { 2 - 8 } & $\begin{array}{c}\text { Basar e Bhat } \\
(2004)^{4}\end{array}$ & $-7,503$ & - & 0,36 & - & - & - \\
\cline { 2 - 8 } & $\begin{array}{c}\text { Blackstone et } \\
\text { al (2006) }\end{array}$ & \multicolumn{2}{|c|}{0,1151} & \multicolumn{2}{c|}{0,1726} & & - \\
\cline { 2 - 8 } & $\begin{array}{c}\text { Loo (2008) } \\
\end{array}$ & $-0,0189$ & $-0,0183$ & 0,0161 & 0,0157 & $-0,0002$ & $-0,0004$ \\
\cline { 2 - 8 } & $\begin{array}{c}\text { Ishii et al } \\
(2009)^{7}\end{array}$ & $-0,0815$ & $-0,0774$ & 0,4168 & 0,6322 & $-0,0077$ & $-0,0259$ \\
\cline { 2 - 8 } & $\begin{array}{c}\text { De Luca } \\
(2012)^{8}\end{array}$ & - & $-0,72$ & - & 0,22 & - & $-0,62$ \\
\hline
\end{tabular}

\footnotetext{
${ }^{1}$ Fonte: autores, com base em levantamento bibliográfico.

${ }^{2}$ Resultados do modelo que considera tempo de acesso linear e frequência não linear. $\mathrm{R}^{2}$ é igual a 0,756 para passageiros a negócio e igual a 0,61 para passageiros que não viajam a negócio.

${ }^{3}$ Resultados do modelo para a base de dados do mês de agosto e que considera frequência não linear. O custo de acesso também foi incluído no modelo.

${ }^{4}$ Resultados do modelo logit multinomial com formação probabilística de painel de alternativas. Outras variáveis compostas pela combinação do tempo de acesso e da frequência com renda, sexo e características da viagem foram incluídas no modelo.

${ }^{5}$ Resultados do modelo probit multivariado para o aeroporto John F. Kennedy. Não considera tempo de acesso, mas distância da residência ao aeroporto (estatisticamente significante a 10\%). A proxy para frequência é a disponibilidade de voos internacionais (estatisticamente significante a 1\%). Outras variáveis também resultaram estatisticamente significantes a 1\%: experiência com outro aeroporto e disponibilidade de estacionamento.

${ }_{7}^{6}$ Resultados do modelo logit multinomial com variáveis estatisticamente significantes a 5\%.

${ }^{7}$ Resultados do modelo logit condicional, o qual engloba outras variáveis: atrasos no pouso e na decolagem e tarifas para diferentes grupos de renda.

${ }^{8}$ Resultados do modelo logit multinomial para voos diretos com as variáveis frequência e tempo de acesso não lineares (transformação logarítmica e Box-Cox, respectivamente). Para este modelo, o $\mathrm{R}^{2}$ é igual a 0,342.
} 


\section{Conclusão}

A análise da competição em sistemas de múltiplos aeroportos representa um tema bastante discutido na literatura, em função de sua importância para o planejamento aeroportuário e das companhias aéreas. Verifica-se que a maior parte dos estudos nessa área utilizam modelos de escolha discreta baseados na teoria da utilidade para simular o comportamento do usuário de transporte aéreo diante de diferentes alternativas. Alguns modelos são mais simples, englobando apenas uma dimensão (escolha do aeroporto propriamente dita), ao passo que outros tentam representar a realidade de forma mais fiel, incluindo outras dimensões no problema, como a escolha do modal de acesso ao aeroporto e da companhia aérea. Apesar disso, a maioria das conclusões obtidas converge para o fato de que a frequência de voo, o tempo de acesso e a tarifa são os atributos mais importantes para o passageiro.

As perspectivas futuras nessa área envolvem o desenvolvimento de novos modelos de escolha aeroportuária, com validação e comparação dos mesmos com os modelos de escolha discreta, bem como o aumento do número de dimensões analisadas no problema, a fim de se aproximar cada vez mais da situação real. É importante observar que alguns parâmetros são raramente incluídos na modelagem, tais como a escolha do aeroporto de chegada e do dia da viagem.

\section{Referências}

Basar, G. e Bhat, C. R. (2004) A parameterized consideration set model for airport choice: an application to the San Francisco Bay area. Transportation Research Part B, vol. 38, n. 10, pp. 889-904.

Blackstone, E. A., Buck, A. J. e Hakim, S. (2006) Determinants of airport choice in a multi-airport region. Atlantic Economic Journal, vol. 34, pp. 313-326.

Carlsson, F. (2010) Design of stated preference surveys: is there more to learn from behavioral economics? Environmental and Resource Economics, vol. 46, n. 2, pp. 167-177.

Collins, A. T., Rose, J. M. e Hess S. (2012) Interactive stated choice surveys: a study of air travel behaviour. Transportation Research, vol. 39, pp. 55-79.

De Luca, S. (2012) Modelling airport choice behaviour for direct flights, connecting flights and different travel plans. Journal of Transport Geography, vol. 22, pp.148-163.

Edwards, W. (1954) The theory of decision making. Psychological Bulletin, vol. 51, n. 4, pp. 380-417.

Harvey, G. (1987) Airport choice in a multiple airport region. Transportation Research Part A, vol. 21, n. 6, pp. 439-449. 
Hensher, D., Louviere, J. e Swait, J (1998) Combining sources of preference data. Journal of Econometrics, vol. 89, n. 1, pp. 197-221.

Hensher, D. A. e Greene, W. H. (2003) The mixed logit model: the state of practice. Transportation, vol. 30, n. 2, pp. 133-176.

Ishii, J., Jun, S. e Dender, K. V. (2009) Air travel choices in multi-airport markets. Journal of Urban Economics, vol. 65, pp. 216-227.

Loo, B. (2008) Passengers' airport choice within multi-airport regions (MARs): some insights from a stated preference survey at Hong Kong International Airport. Journal of Transport Geography, vol. 16, n. 2, pp. 117-125.

Loomis, B. (2011) What's to know about hypothetical bias in stated preference valuation studies? Journal of Economic Surveys, vol. 25, n. 2, pp. 363-370.

Pels, E., Nijkamp, P. e Rietveld, P. (2003) Access to and competition between airports: a case study for the San Francisco Bay Area. Transportation Research Part A, vol. 37, n. 1, pp. 71-83.

Walker, J. e Ben-Akiva, M. (2002) Generalized random utility model. Mathematical Social Sciences, vol. 43, n. 3, pp. 303-343. 